\title{
SHR/NCrl rats as a model of ADHD can be discriminated from controls based on their brain, blood, or urine metabolomes
}

\author{
Camille Dupuy ${ }^{1}$, Pierre Castelnau ${ }^{1,2}$, Sylvie Mavel', Antoine Lefevre', Lydie Nadal-Desbarats', Sylvie Bodard', \\ Julie Busson ${ }^{1}$, Diane Dufour-Rainfray ${ }^{1,2}$, Helene Blasco ${ }^{1,2}$, Patrick Emond ${ }^{1,2}$ and Laurent Galineau (1)
}

\begin{abstract}
Attention-Deficit Hyperactivity Disorder (ADHD) is one of the most common neurodevelopmental disorder characterized by inattention, impulsivity, and hyperactivity. The neurobiological mechanisms underlying ADHD are still poorly understood, and its diagnosis remains difficult due to its heterogeneity. Metabolomics is a recent strategy for the holistic exploration of metabolism and is well suited for investigating the pathophysiology of diseases and finding molecular biomarkers. A few clinical metabolomic studies have been performed on peripheral samples from ADHD patients but are limited by their access to the brain. Here, we investigated the brain, blood, and urine metabolomes of SHR/NCrl vs WKY/NHsd rats to better understand the neurobiology and to find potential peripheral biomarkers underlying the ADHD-like phenotype of this animal model. We showed that SHR/NCrl rats can be differentiated from controls based on their brain, blood, and urine metabolomes. In the brain, SHR/NCrl rats displayed modifications in metabolic pathways related to energy metabolism and oxidative stress further supporting their importance in the pathophysiology of ADHD bringing news arguments in favor of the Neuroenergetic theory of ADHD. Besides, the peripheral metabolome of SHR/NCrl rats also shared more than half of these differences further supporting the importance of looking at multiple matrices to characterize a pathophysiological condition of an individual. This also stresses out the importance of investigating the peripheral energy and oxidative stress metabolic pathways in the search of biomarkers of ADHD.
\end{abstract}

\section{Introduction}

Attention-deficit/hyperactivity disorder (ADHD) is one of the most common neurodevelopmental disorder affecting $5.3 \%$ of school-aged children ${ }^{1}$ and $2.5-4.9 \%$ of adults $^{2,3}$. ADHD symptoms include inattention and hyperactivity/impulsivity, with three symptom presentations described by the Diagnostic and Statistical Manual of Mental Disorders, Fifth Edition (DSM-5): predominantly inattentive, predominantly hyperactive/ impulsive, and combined profiles, which may vary over time. The diagnosis mainly relies on detailed clinical

Correspondence: Laurent Galineau (galineau@univ-tours.fr)

'UMR 1253, iBrain, Université de Tours, Inserm, Tours, France

${ }^{2} \mathrm{CHRU}$ Tours, Tours, France

These authors contributed equally: Patrick Emond, Laurent Galineau evaluations of the symptoms and careful assessment of the attention and executive functions $\mathrm{s}^{4}$. However, the high heterogeneities of the phenotypic presentations and temporal courses of the disorder, along with the numerous comorbidities, render this diagnosis difficult to establish and often delayed ${ }^{5}$. Besides, false positives and false negatives are not unusual. Thus, there is a crucial need for biomarkers to facilitate an early and reproducible ADHD diagnosis in order to promote appropriate care and follow-up of the patients. Toward this aim, numerous genetic and neuroimaging studies were conducted in ADHD patients over the past two decades but failed to provide relevant tools for reliable diagnosis on a routine basis ${ }^{4}$. 
To date, metabolomic strategies remain largely unexplored in the search for ADHD biomarkers ${ }^{6}$. Only a few hypothesis-driven and one nonhypothesis-driven studies using various sample types have been performed in ADHD patients. Therefore, additional studies are needed to identify useful peripheral biomarkers ${ }^{7,8}$. Metabolomics aims at providing qualitative and quantitative changes of hundreds of metabolites ${ }^{9}$ and has been increasingly used to identify molecular fingerprints in neurodevelopmental disorders. In this context, metabolomic studies in animal models are of a great interest as it allows to access peripheral but also central compartments to better evaluate how potential peripheral metabolomic changes can reflect central modifications.

The goal of the present study was to explore the cerebral and peripheral metabolomes of SHR/NCrl vs WKY/ NHsd rats, which is one of the most extensively studied and characterized animal model of ADHD with good face and construct validities ${ }^{10-15}$. Our specific objectives were (i) to characterize the modifications in the brain, blood, and urine metabolomes in this animal model of ADHD, and (ii) to identify peripheral modifications in metabolites/metabolic pathways related to the reported cerebral changes.

\section{Materials and methods Subjects}

The subjects were eight male SHR/NCrl rats (Charles River, Germany), which were compared to eight male WKY/NHsd control rats (Harlan, United Kingdom); all rats were 8 weeks old, i.e., before the onset of hypertension in the SHR/NCrl rats, as described by Sagvolden et al. ${ }^{11}$. Animals were housed in groups of 2 or 3 under humidity-and temperature-controlled conditions and a 12:12 light-dark cycle (light on at 7:00 AM) with ad libitum access to food and water. All animal experiments were conducted in accordance with EU guidelines (EU Directive 2010/63/EU for animal experiments), and approved by a local Ethics Committee (Comité d'Ethique en Expérimentation Animale-Pays de la LoireFrance).

\section{Sample collection}

Urine samples were collected before sacrifice by handling the animals. Immediately after decapitation, blood samples were collected in vials filled with $1.8 \mathrm{mg}$ of EDTA (Sigma-Aldrich, France), centrifuged at $11,500 \times g$ and $4{ }^{\circ} \mathrm{C}$ for $10 \mathrm{~min}$ and the plasma retrieved. Samples were stored at $-80^{\circ} \mathrm{C}$. The brains were quickly removed and prepared as described below.

\section{Sample preparation}

Brain samples were freeze-dried (FreeZone ${ }^{\circledR} 4.5 \mathrm{~L}$, Labconco, USA) at $-107^{\circ} \mathrm{C}$ and 0.2 mbar for $12 \mathrm{~h}$. The lyophilized material $(3 \mathrm{mg}$ ) was mixed with $750 \mu \mathrm{L}$ of $\mathrm{ACN} / \mathrm{H}_{2} \mathrm{O}(1: 1)$, vortexed for $15 \mathrm{~s}$, homogenized by gentle planar shaking at $4{ }^{\circ} \mathrm{C}$ for $30 \mathrm{~min}$ and centrifuged at $11,500 \times g$ and $4{ }^{\circ} \mathrm{C}$ for $15 \mathrm{~min}$ (Thermo Fischer, USA). Supernatants $(500 \mu \mathrm{L})$ were concentrated under vacuum (SpeedVac, Thermo Fischer, USA) at $45^{\circ} \mathrm{C}$ for $4 \mathrm{~h}$. The resultant dry residues were reconstituted in $100 \mu \mathrm{L}$ of $\mathrm{MeOH} / \mathrm{H}_{2} \mathrm{O}$ (1:9).

Urine samples were diluted to the tenth with ultrapure water and vortexed for $15 \mathrm{~s}$.

Metabolites were extracted from $100 \mu \mathrm{L}$ of plasma by adding $800 \mu \mathrm{L}$ of $\mathrm{MeOH} / \mathrm{H}_{2} \mathrm{O}$ (1:1). The samples were then vortexed for $15 \mathrm{~s}$, homogenized by gentle planar shaking at $4{ }^{\circ} \mathrm{C}$ for $30 \mathrm{~min}$, and centrifugated at $11,500 \times g$ and $4{ }^{\circ} \mathrm{C}$ for $10 \mathrm{~min}$ to collect $500 \mu \mathrm{L}$ of supernatant. Supernatants were concentrated under vacuum (SpeedVac, Thermo Fischer, USA) at $45^{\circ} \mathrm{C}$ for $4 \mathrm{~h}$. The dried residues were reconstituted in $100 \mu \mathrm{L}$ of $\mathrm{MeOH} / \mathrm{H}_{2} \mathrm{O}$ (1:9). Each prepared sample was then transferred to a 96well plate for liquid chromatography-high resolution mass spectrometry (LC-HRMS) analysis. Quality control samples (QCs) were obtained from a pooled mixture of equal volumes of all samples for each matrix. Fifteen QCs were injected to equilibrate the chromatographic system before each analytic batch and QCs were analyzed every 10 samples.

\section{Liquid Chromatography-High Resolution Mass Spectrometry Analysis (LC-HRMS)}

LC-HRMS analysis was performed on a UPLC Ultimate 3000 system (Dionex, Germany) coupled to a Q-Exactive mass spectrometer (Thermo Fisher, Germany) and operated in positive and negative electro spray ionization (ESI + and ESI-). Chromatography was performed as previously described ${ }^{16}$. For both modes, heated ESI source parameters consisted in a spray voltage of $3 \mathrm{kV}$, a capillary temperature of $380^{\circ} \mathrm{C}$, a heater temperature of $350^{\circ} \mathrm{C}$, a sheath gas flow of 40 arbitrary units (AU), an auxiliary gas flow of $20 \mathrm{AU}$, a spare gas flow of $2 \mathrm{AU}$, and a tube lens voltage of $50 \mathrm{~V}$. The full-scan acquisition ranged from 58 to $870 \mathrm{~m} / z$ and the instrument operated at 70,000 resolution $(m / z=200)$, with an automatic gain control target of $1 \times 10^{6}$ charges and a maximum injection time of $250 \mathrm{~ms}$. The identity of each sample was processed by experimenters blinded its experimental group.

\section{Data processing for targeted LC-HRMS}

A library of 495 standard metabolites (Mass Spectroscopy Metabolite Library, IROA technologies, USA) was used for the construction of an in-house metabolite database. Targeted molecules from our database were selected and integrated into Xcalibur 2.2 (Thermo Fisher, USA) as previously described ${ }^{17}$. Each peak area was normalized to the total of peak areas of interest. Metabolites 


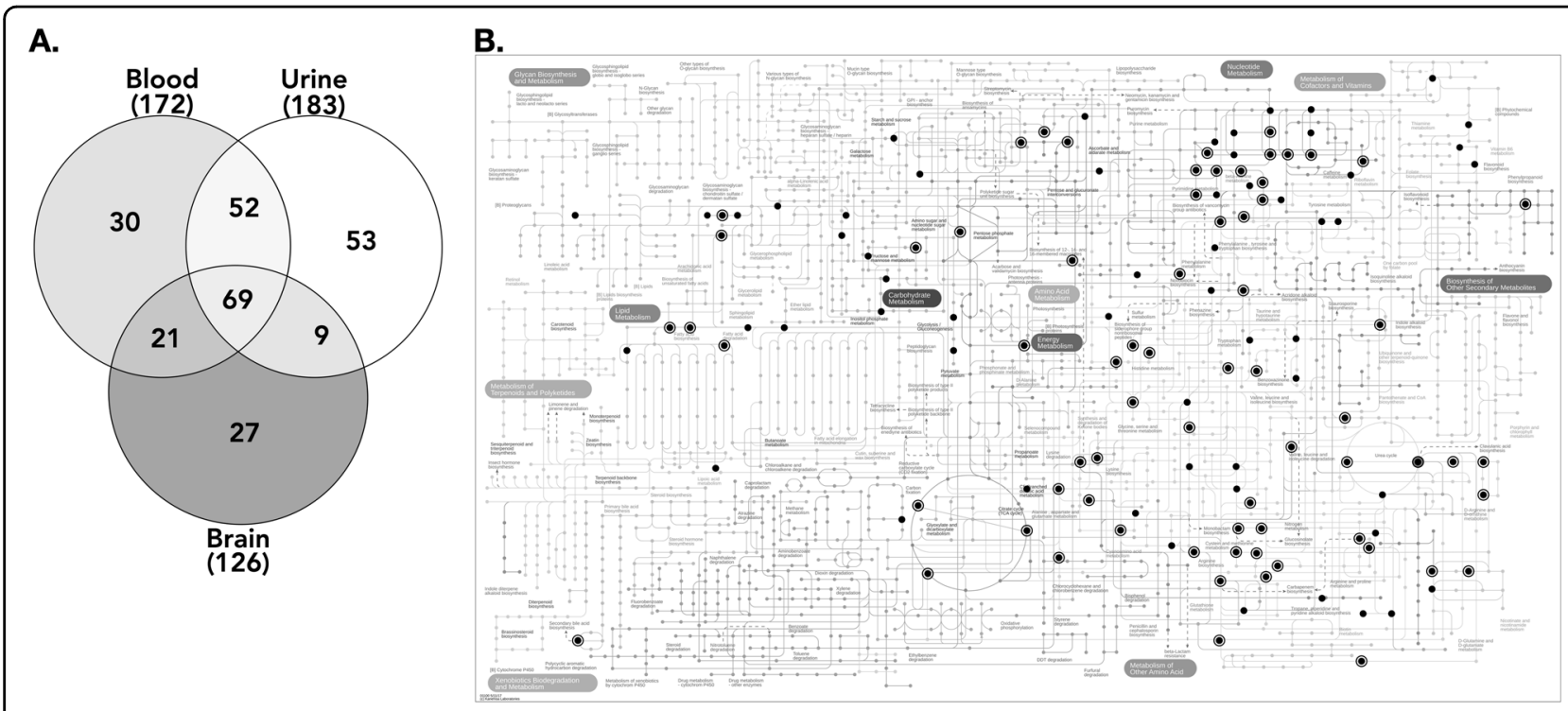

Fig. 1 Number of metabolites detected in the brain, blood, and urines of SHR/NCrl and WKY/NHsd rats. A Venn diagram presenting the number of metabolites detected in each compartment. B Black circles representing metabolites detected in the blood or urine compartments of $\mathrm{SHR} / \mathrm{NCrl}$ and WKY/NHsd rats. The open circles correspond to the metabolites detected in the brain of SHR/NCrl and WKY/NHsd rats.

detected with greater than $30 \%$ variance $(\mathrm{CV} \%)$ in $\mathrm{QC}$ samples were not selected for further analysis.

\section{Statistical analysis}

The metabolic card was performed using the web-based tool iPath 3.0 to represent all the metabolites detected in the brain on the metabolic pathways based on the latest version of the Kyoto Encyclopedia of Genes and Genomes (KEGG, www.kegg.jp). A Venn diagram was performed to assess the complementarity between the rat's brain, plasma, and urines samples using the jvenn online tool (http://jvenn.toulouse.inra.fr/app/index.html).

Multivariate analyses were performed using Metaboanalyst $4.0^{18}$, and included principal component analyses (PCA) and partial least squares discriminant analyses (PLS-DA). PCA were performed on brain, plasma, and urine samples to identify outliers (one rat from each group of the brain samples was excluded), and to perform unsupervised clustering of SHR/NCrl and WKY/NHsd rats. PLS-DA were performed on brain, plasma, and urine samples to build discriminant models between the two strains. Each model was characterized by the cumulative modeled variation in the $\mathrm{Y}$ matrix $R^{2} Y$, and the cross-validated predictive ability $Q^{2}$ values, providing the discriminant metabolites called variable importance in projection (VIP). Metaboanalyst 4.0 was also used for univariate analyses using volcano plots on brain, plasma, and urine samples to select the metabolites whose levels were statistically significantly different between SHR/NCrl and WKY/NHsd rats ( $p$-value corrected for False Discovery Rate $=[\mathrm{FDR}]$ values), and whose concentrations differed between SHR/NCrl rats and controls by less than 0.75 -fold or more than 1.25 fold. Pathway analyses using Metaboanalyst 4.0 were conducted on the VIP metabolites. The pathways were considered when displaying FDR values $<0.05$ and a non-null impact value. Correlation analyses were performed for metabolites that were significantly altered in the brain and plasma and/or urines samples using Metaboanalyst 4.0 and were considered significant when $p<0.05$. Linear regression curves were generated using GraphPad Prism 7 (GraphPad Prism, USA).

\section{Results}

Metabolic profile of the central and peripheral compartments of SHR/NCrl and WKY/NHsd rats

Starting from a library of 495 compounds, the number of metabolites detected in both rat strains ranged from 125 to 182 , representing $25-37 \%$ of the whole library (Fig. 1A). The number of metabolites detected in the brain was slightly smaller compared to the peripheral compartments (125 metabolites in the brain vs 171 and 182 in blood and urine, respectively; Fig. 1A). In addition, the brain shared 77 metabolites (61\%) with the urine compartment, and 89 (71\%) with the blood compartment (Fig. 1A). Finally, 68 metabolites (55\% of the cerebral metabolites) were detected in all the matrices (Fig. 1A). Based on pathway analyses performed on each dataset, the detected metabolites were involved in 47 , 50, and 51 metabolic pathways, respectively, described in the rat urine, blood, and brain compartments (Fig. 1B). 

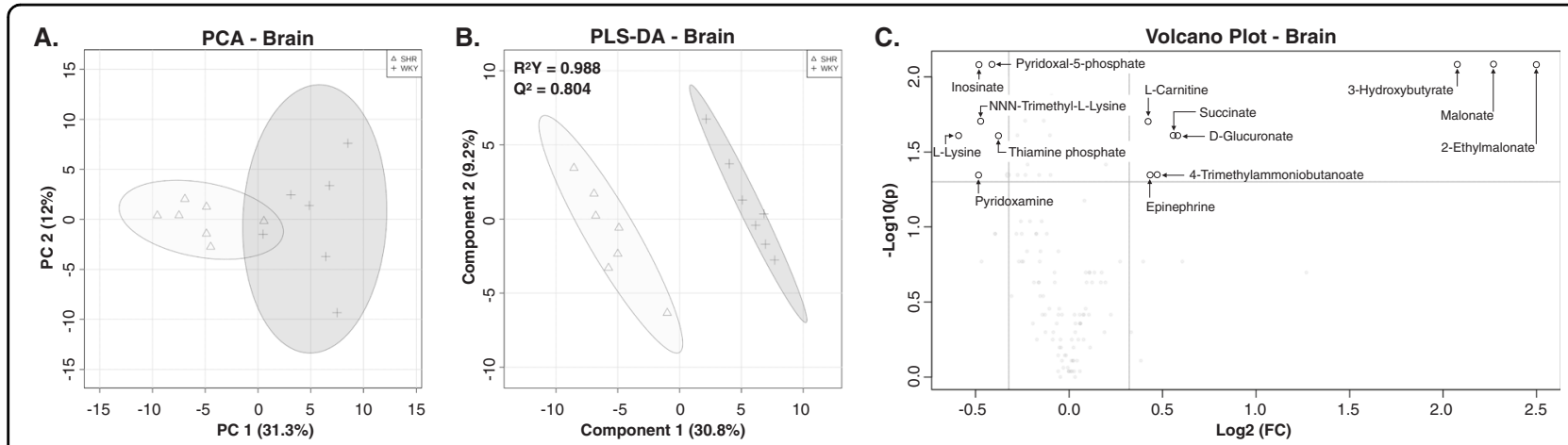

Fig. 2 Distinction between the SHR/NCrl and WKY/NHsd rats ( $\boldsymbol{n}=\mathbf{7}$ per group) based on brain metabolome. A Principal component analysis (PCA) score plot differentiating two groups corresponding to the brain metabolomes of SHR/NCrl (triangles) and WKY/NHsd (crosses) rats. B Partial least squares discriminant analysis (PLS-DA) showing the discriminant model based on two components built to separate SHR/NCrl (triangles) from WKY/NHsd brain metabolomes (crosses). C Volcano plot showing the metabolites with significant FDR values between SHR/NCrl and WKY/NHsd rats and fold differences lower than 0.75 or greater than 1.25 .

SHR/NCrl rats can be differentiated from controls based on their cerebral metabolome

Applying a PCA to the 125 metabolites detected in the brain enabled to clearly differentiate the SHR/NCrl rats from controls using two components representing $43 \%$ of their overall variations between the two strains (Fig. 2A). In addition, the distance between the subjects in the SHR/ $\mathrm{NCrl}$ group suggests a low interindividual variability of their brain metabolome (Fig. 2A). The SHR/NCrl rats were separated from controls using a PLS-DA analysis based on a model showing an accuracy of 0.929 , a $R^{2} Y$ value of 0.988 , and a predictive ability $\left(\mathrm{Q}^{2}\right)$ of 0.804 for the two first components (Fig. 2B). Fifty-three metabolites showed variable importance in prediction (VIP) scores superior to 1 (Table S1). Among them, 31 displayed significantly different brain concentrations in SHR/NCrl rats vs controls (Table S1). Of these 31 metabolites, 14 had concentrations that differed between SHR/NCrl rats and controls by less than 0.75 -fold or more than 1.25-fold (Fig. 2C, Table S1). In detail, the brain concentrations of 2-ethylmalonate, malonate, 3-hydroxybutyrate, glucuronate, succinate, 4trimethylammoniobutanoate, epinephrine, and carnitine were significantly higher in SHR/NCrl rats vs controls, while the concentrations of lysine, pyridoxamine, inosinate, NNN-trimethyl-lysine, pyridoxal-5-phosphate, and thiamine phosphate were significantly lower (Table S1). A pathway analysis performed using the 31 significantly different metabolites revealed modifications in 14 metabolic pathways between SHR/NCrl and control animals (Table 1). Among them, five pathways were related to amino acids metabolism (alanine and beta-alanine metabolism; aspartate and glutamate metabolism; lysine degradation; arginine and proline metabolism and tyrosine metabolism), four to energy metabolism (pantothenate and CoA biosynthesis; butanoate metabolism; citrate cycle and pentose and glucuronate interconversions), three to nucleotide metabolism (purine, pyrimidine and nicotinate metabolisms), and two to vitamin metabolism (vitamin B6 and ascorbate metabolisms) (Table 1). This pathway analysis did not include five metabolites of the VIPs, which are also involved in energy (2-ethylmalonate, malonate, 3-hydroxybutyrate, and carnitine), and vitamin (thiamine phosphate) metabolisms.

\section{SHR/NCrl rats can also be differentiated from controls based on their peripheral metabolomes}

Applying a PCA to the metabolites detected in the blood, and urine enabled to clearly differentiate the SHR/NCrl rats from the controls (Fig. 3A, D). Based on the metabolites detected in the blood, the PCA analysis differentiated the SHR/NCrl rats $v s$ controls using two components representing $44 \%$ of the overall variation between the two strains (Fig. 3A). Similarly, the PCA based on the metabolites detected in the urine compartment enabled to easily divide the animals into two distinct populations corresponding to SHR/NCrl and control rats (Fig. 3D). This was achieved using two components representing $60 \%$ of the overall variation existing between the two strains (Fig. 3D).

The SHR/NCrl rats were also differentiated from controls using a PLS-DA based on blood and urine metabolites (Fig. 3B, E). The model obtained on the blood metabolites had an accuracy of $1.0, \mathrm{a} \mathrm{R}^{2} \mathrm{Y}$ value of 0.989 , and a $\mathrm{Q}^{2}$ of 0.913 for two components (Fig. 3B). The model obtained on urine metabolites showed an accuracy of 1.0, a $R^{2} Y$ value of 0.986 , and a $Q^{2}$ of 0.944 for two components (Fig. 3E). Sixty-seven VIPs were observed for the blood compartment including 41 metabolites with statistically significant distinct concentrations between SHR/NCrl rats and controls (Table S2). A total of 105 metabolites (including 90 VIPs) showed significantly different urine concentrations between $\mathrm{SHR} / \mathrm{NCrl}$ rats and controls (Table S3). 


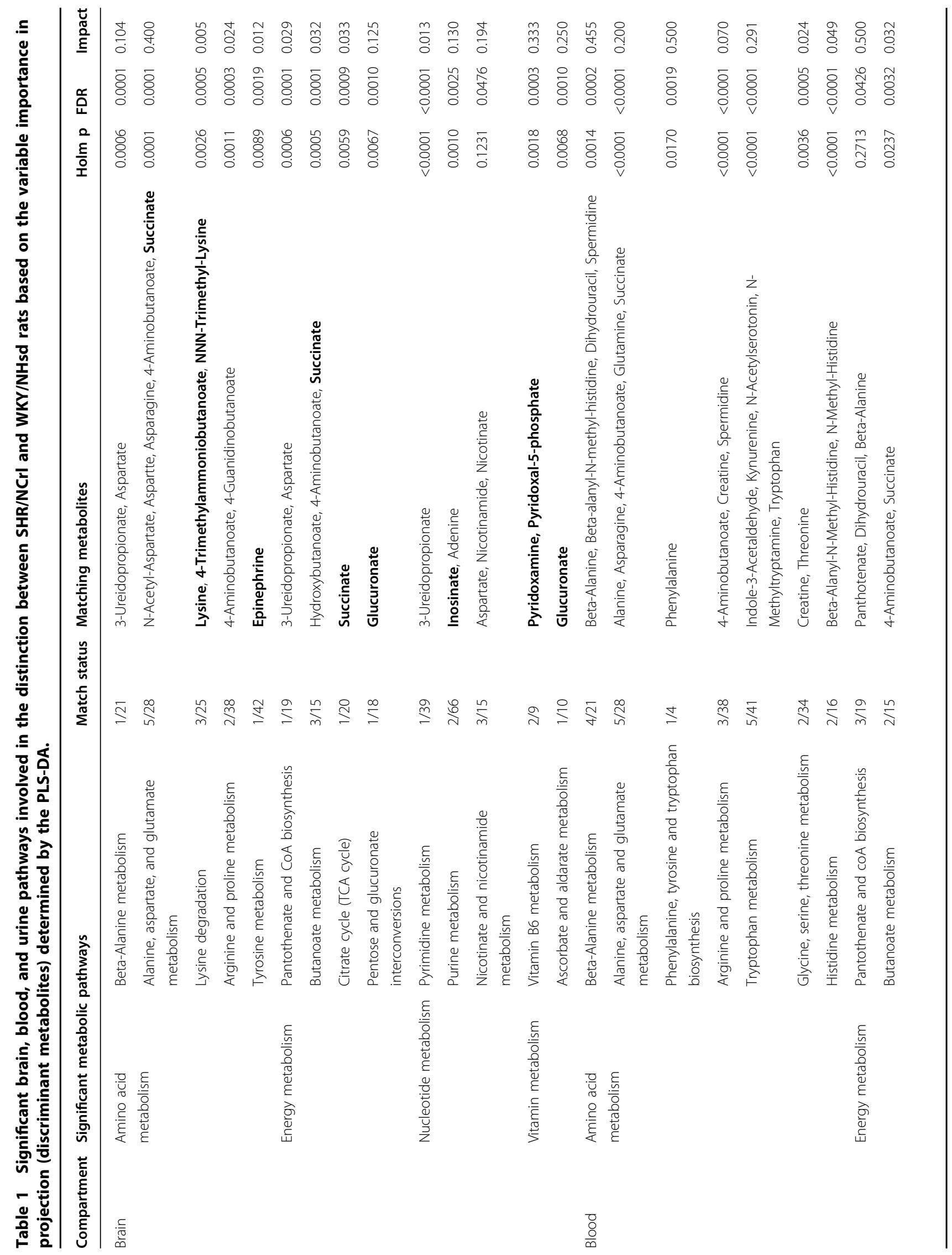




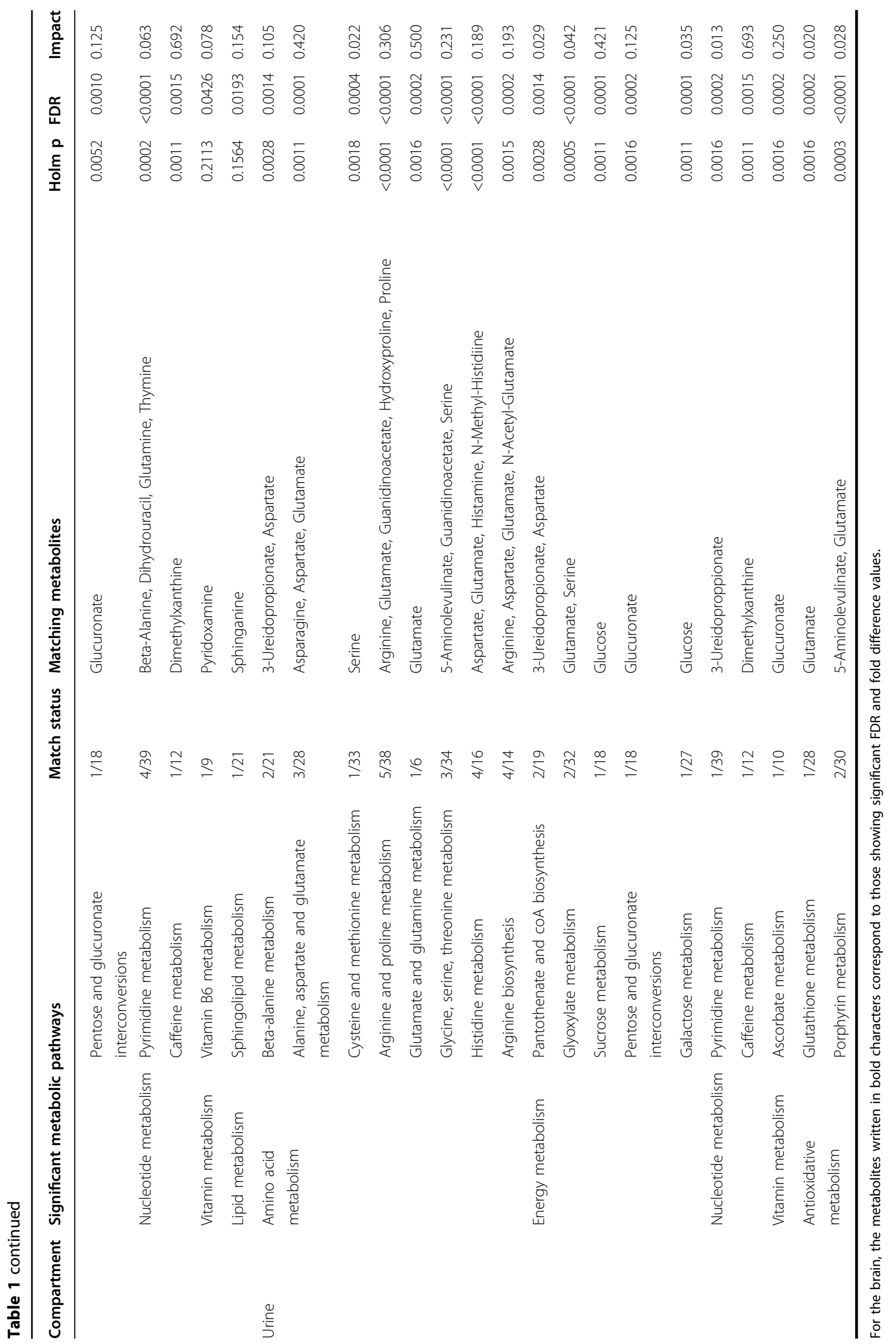




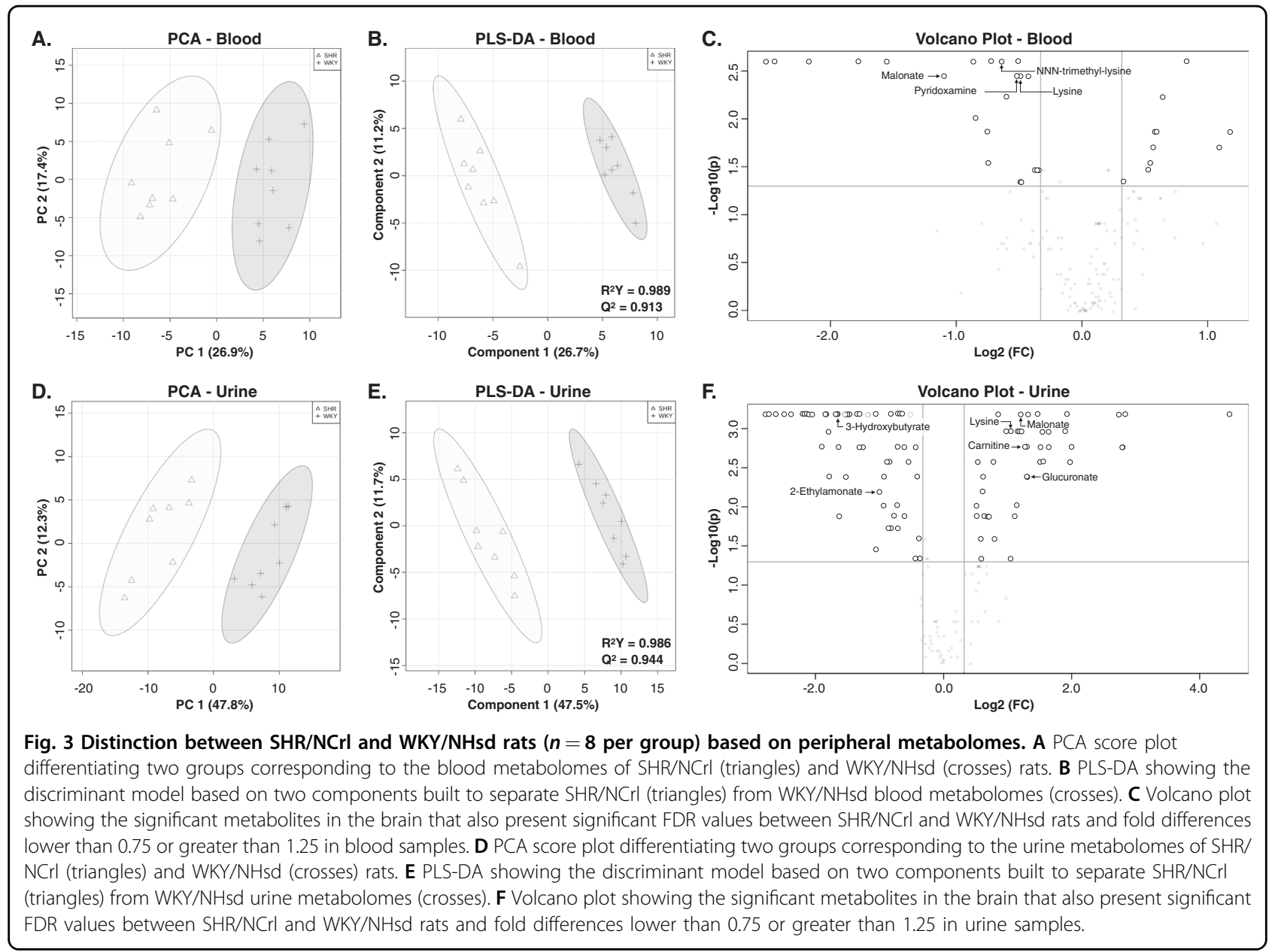

The pathway analysis showed significant modifications in 14 and 18 metabolic pathways in blood and urines of SHR/NCrl rats, respectively (Table 1). Interestingly, more than half of the pathways detected in the brain were also observed in the periphery (i.e., significant changes in 8 of the 14 brain pathways were found in the periphery; Fig. 4B). Thus, SHR/NCrl rats showed significant modifications in the amino acids, energy, nucleotides, and vitamin metabolisms in all the peripheral compartments as observed in the brain, as well as changes in the lipid metabolism in the blood and antioxidant metabolism in urines (Table 1).

Only looking at metabolites with significant FDR and fold-change values between SHR/NCrl and WKY/NHsd rats, six metabolites were significantly modified in both brain and urine compartments, and four were significantly modified in both brain and blood compartments. Malonate and lysine concentrations between SHR/NCrl and WKY/NHsd rats were different in brain, blood, and urines. Changes in pyridoxamine and NNNtrimethyl-lysine between SHR/NCrl vs WKY/NHsd rats were specific to the brain and blood compartments, while changes in 2-ethylmalonate, 3-hydroxybutyrate, carnitine, and glucuronate were specific to brain and urines compartments.

\section{Relationships between the brain and peripheral metabolomes}

A hierarchical clustering revealed that the compartment has more impact than the strain on the metabolomic differences observed, with a clear distinction of the central vs peripheral metabolomes (Fig. 4A). Significant correlations between brain and peripheral concentrations of gluruconate and malonate were specifically observed in SHR/NCrl rats (Fig. 4B). A significant negative correlation between the levels of glucuronate in the brain and in the urines $\left(\mathrm{r}^{2}=0.849, p=0.002\right)$ (Fig. 4B). In addition, malonate concentrations in brain and urines were positively correlated to each other in SHR/NCrl rats $\left(\mathrm{r}^{2}=0.815\right.$, $p=0.026)$ (Fig. 4B).

\section{Discussion}

This study provides the first metabolomic characterization of the ADHD model based on the comparison of 


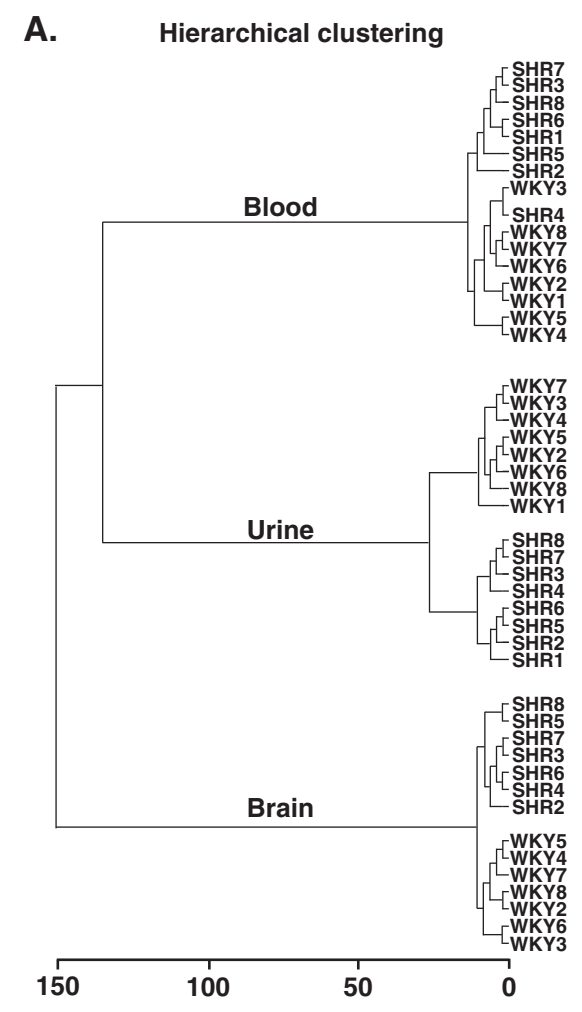

B.
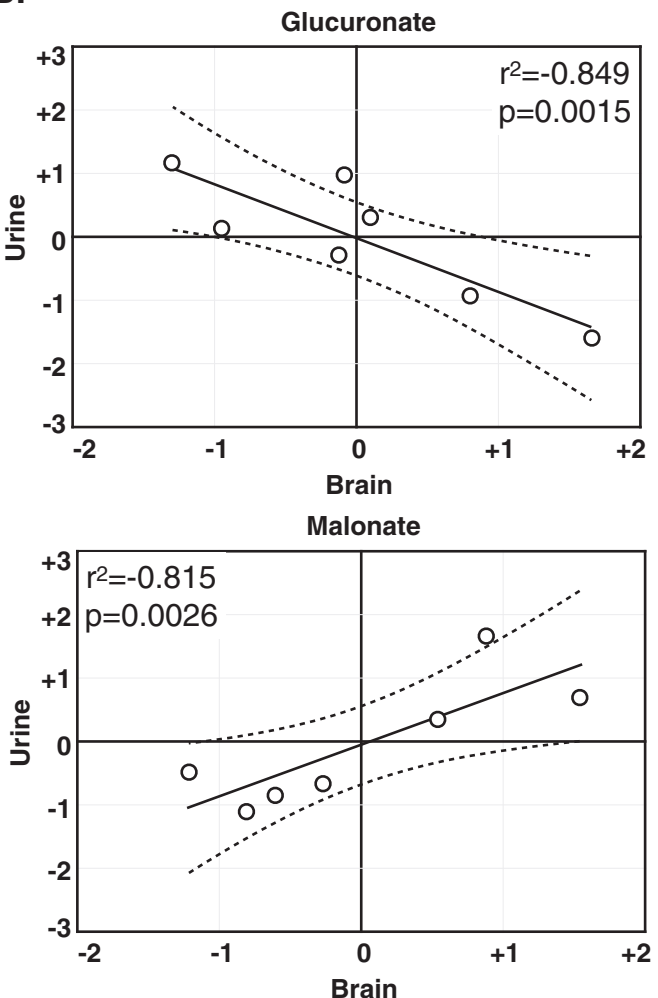

Fig. 4 Relationships between the brain and peripheral metabolomes. A Hierarchical clustering analysis showing differences between brain and peripheral metabolomes in SHR/NCrl and WKY/NHsd rats. B Correlation between brain and peripheral concentrations of glucuronate and malonate in $\mathrm{SHR} / \mathrm{NCrl}$ rats.

$\mathrm{SHR} / \mathrm{NCrl}$ rats vs WKY/NHsd animals used as controls. Here we extensively studied the differences existing between SHR/NCrl rats vs controls in the brain and in the peripheral matrices. We showed that the brain, blood, and urine metabolomes can be used to discriminate SHR/ $\mathrm{NCrl}$ rats from controls. The main cerebral differences between the two strains mainly consist in modifications in amino acid, energy, and vitamin metabolisms, that were also observed in blood and urines. In addition, malonate and glucuronate concentrations in the brain and urine compartments of $\mathrm{SHR} / \mathrm{NCrl}$ rats were significantly correlated.

\section{Limitations of the animal model}

Even though SHR compared to WKY rats are one of the mostly used animal model of ADHD, it has also been criticized for its face and predictive validities, based on studies showing no differences in locomotor activity between SHR rats and controls, and others suggesting a lack of effect of methylphenidate on the ADHD-related phenotypes of this animal model ${ }^{19-23}$. Sagvolden and collaborators reported genetic and behavioral differences in existing among the strains of SHR and WKY rats, and showed the importance of using specifically SHR rats from Charles River Germany (SHR/NCrl) compared to WKY rats from Envigo U.K. (WKY/NHsd) to model the ADHD-related inattention, impulsivity, and hyperactivity ${ }^{11}$. As also highlighted by Sagvolden and collaborators, most of the studies questioning the face and predictive validities of SHR/NCrl vs WKY/NHsd rats as a model of ADHD were confounded by the settings used to assess rat's behaviors ${ }^{11}$. In this study, we used the recommended strains of SHR and WKY rats to match with the studies that extensively characterized the ADHDrelated behaviors of the animal model.

This animal model of ADHD is also confounded by the development of hypertension from 10 to 12 weeks of age $^{24}$, and cognitive deficits observed in 11-week-old animals $^{25}$. A recent metabolomic study performed in urine and plasma samples of 40-weeks-old SHR and WKY rats described a significant correlation between urine and blood variations of several metabolites and hypertension ${ }^{26}$ (urine levels of dimethylarginine, N2-acetyl-L-ornithine, buthionine sulfoximine, uric acid, L-isoleucine, and creatinine; blood levels of Vitamine $\mathrm{E}$ and phospholipids). Although we cannot completely rule out that our metabolic data could be related to the development of hypertension in our experimental conditions, we worked on 
8-weeks-old animals, and our peripheral data are not in line with the urinary and plasma biomarkers significantly correlated with hypertension in these studies.

\section{SHR/NCrl rats mainly differed from controls through their cerebral energy and adrenaline metabolisms}

SHR/NCrl rats showed lower brain levels in thiamine phosphate compared to controls. Interestingly, old reports suggested that vitamin supplementations, including thiamine, could carry beneficial effects on ADHD symptoms ${ }^{27,28}$. Thiamine phosphate is a cofactor of the pyruvate dehydrogenase, alpha-ketoglutarate dehydrogenase, and trans-ketolase ${ }^{29}$, involved in the glycolysis, pentose phosphate pathway and Krebs cycle. Therefore, significant alterations of the cerebral energy metabolism were expected in this ADHD animal model. This is consistent with studies reporting decreased expression of the dihydrolipoyl dehydrogenase, a part of the pyruvate dehydrogenase complex in the medulla oblongata of SHR/ $\mathrm{NCrl}$ rats $^{30}$, along with decreased ATP production capacities in discrete brain regions in this animal model of $\mathrm{ADHD}^{30,31}$.

In line with such hypothesis, we observed significantly elevated levels in succinate, malonate and 2ethylmalonate in SHR/NCrl rats compared to controls. Both malonate and 2-ethylmalonate inhibit several Krebs cycle's enzymes, including the succinate dehydrogenase $^{32,33}$ fitting well with the higher levels of succinate detected. Based on the key role of succinate in the Krebs cycle and in the respiratory chain, these results further support a dysfunctional brain energy metabolism in this animal model of ADHD. In addition, elevated succinate levels and mitochondrial dysfunctions have been associated to an increased oxidative stress, which has been extensively described in the brain of this animal model $^{34,35}$, and in ADHD patients ${ }^{36-39}$. The higher brain levels of 2-ethylmalonate also observed in SHR/NCrl rats are consistent with an increased oxidative stress as it is a reliable lipid peroxidation biomarker ${ }^{40}$. Thus, the higher brain levels of glucuronate detected could reflect an elevated activity of the pentose phosphate pathway due to the increased oxidative stress, as the pentose phosphate pathway is used by neurons to regenerate glutathione ${ }^{41}$. In line with these results and other studies reporting protective effects of methylphenidate on oxidative stress ${ }^{37,38}$, Fagundes and collaborators already showed that a methylphenidate treatment increases the succinate dehydrogenase and activates the mitochondrial respiratory chain of young Wistar rats further supporting our hypothesis ${ }^{42}$.

We also detected higher carnitine and 3hydroxybutyrate brain levels in SHR/NCrl rats vs controls. Carnitine plays a key role in energy metabolism by transporting long-chain fatty acids to the mitochondria for the production of acetyl-CoA via the beta-oxidation, and removes short and medium chain fatty acids formed by metabolic processes to prevent their accumulation in the mitochondria ${ }^{43}$. Thus, these higher brain carnitine levels could reflect modifications in the lipid metabolism in SHR/NCrl rats. Fatty acids and ketone bodies such as 3-hydroxybutyrate are alternative energy substrates during development or when glucose availability is limited ${ }^{31}$. In our experimental conditions, the cerebral glucose concentrations in the SHR/NCrl rats were not different from controls. Moreover, 3-hydroxybutyrate is no longer a substitute of glucose for brain energy metabolism after 30 days of life in rats ${ }^{44}$. Studies reported a switch towards other energy substrates to maintain the Krebs cycle supply with acetyl-CoA in conditions of decreased pyruvate dehydrogenase activity ${ }^{45-47}$. Based on the lower thiamine levels we observed in SHR/NCrl rats, and on its role as cofactor of enzymes including the pyruvate dehydrogenase, we cannot exclude that the higher carnitine and 3-hydroxybutyrate levels we observed could reflect such a switch in energy substrates. Another explanation could rely in their antioxidant properties ${ }^{43,48-50}$ in response to the higher oxidative stress detected in this animal model of $\mathrm{ADHD}^{34,35}$.

Intracerebral injections of malonate have been used as an inducer of energy mitochondrial metabolic dysfunctions, and have been shown to induce modifications in dopamine neurotransmission ${ }^{51}$. Based on the implication of dopamine in the pathophysiology of ADHD, this support that such abnormalities related to neuroenergetic ${ }^{52-54}$ and oxidative stress could be one of the molecular mechanism involved in the pathophysiology of ADHDrelated phenotypes in SHR/NCrl vs WKY/NHsd rats. Thus, we expected to see modifications in the cerebral levels of various neurotransmitters in SHR/NCrl rats vs controls, as the implication of multiple neurotransmitters has already been suggested in $\mathrm{ADHD}^{55-57}$, and dopamine along with noradrenaline modifications have already been described in discrete regions in this animal model ${ }^{13}$. The fact that we analyzed the whole brain rather than discrete regions could explain the lack of results related to neurotransmitters metabolism in our experiments. Instead, we observed the lower brain levels of pyridoxaminerelated compounds and higher adrenaline levels at the whole-brain scale in SHR/NCrl rats. Interestingly, lower peripheral levels of pyridoxamine have been reported in ADHD patients, and pyridoxamine supplementation has been suggested to normalize ADHD symptoms ${ }^{58-60}$. Pyridoxamine derivatives are co-factors of enzymes crucial to the biosynthesis of multiple neurotransmitters such as GABA, glutamate and monoamines ${ }^{61}$ further supporting that the lower pyrixodamine levels observed in our study could be related to modifications in neurotransmitters contents in this animal model of ADHD. 
Concerning adrenaline, higher brain concentrations have already been reported in the medulla oblongata in this animal model, along with greater expression of the phenylethanolamine $\mathrm{N}$-methyltransferase, which is responsible for adrenaline biosynthesis ${ }^{62-67}$. Because the adrenergic neurons of the medulla oblongata are involved in the regulation of blood pressure, these higher levels were associated with the development of hypertension elicited by adult SHR rats. Even though our study was conducted before the appearance of hypertension in this animal model ${ }^{13}$, we cannot exclude that the higher adrenaline levels we observed could be involved in the hypertension development in these rats. However, adrenergic neurons do not only send axonal projections in areas regulating autonomic functions, but also to the locus coeruleus and ventral tegmental area containing noradrenergic and dopaminergic neurons known to be involved in the pathophysiology of $\mathrm{ADHD}^{47,68}$. Thus, it cannot be excluded that higher adrenaline levels could be related to modifications in dopamine and noradrenaline contents in specific brain regions in this animal model of ADHD.

\section{SHR/NCrl rats also exhibited unique peripheral metabolomes}

In this study, we were able to differentiate SHR/NCrl rats from controls using discriminant models based on blood and urine samples with accuracies and predictive abilities superior to what was obtained in the brain. Besides, $61 \%$ of the metabolites involved in the discriminant model in the brain were also involved in the peripheral models, and almost all these metabolites were significantly different between SHR/NCrl rats and controls in central and peripheral compartments. This good coverage of the brain by the peripheral metabolomes should enable to identify valuable peripheral biomarkers related to the neurobiology underpinning this animal ADHD model. At the scale of metabolomic pathways, the urine compartment shared almost $80 \%$ of the differences detected in the blood. The interest of looking at the urine compartment in the context of neuropsychiatric diseases has already been highlighted, as this compartment is not subjected to the homeostatic changes observed in the blood ${ }^{69}$. Since a large number of metabolites are concentrated in urines, this could result in an enhanced sensitivity for the detection of low metabolic variations. In addition, the easy access to urines in humans makes it a compartment of choice for patients in which blood sampling can be more complex to collect. Thus, this further highlight the complementarity between peripheral compartments, and the interest at looking at multiple peripheral matrices to better reflect a cerebral pathophysiological condition.
As in the brain, our blood and urine results revealed significantly modified pathways related to amino acids, energetic, and vitamin metabolisms along with oxidative stress in SHR/NCrl rats vs controls. Clinical studies already explored peripheral matrices such as plasma, serum, saliva, and urine in ADHD subjects. Most of these studies have examined levels of growth factors, cortisol, melatonin, catecholamines, fatty acids, amino acids, oxidative stress, and pyridoxine metabolisms in ADHD patients, but no reliable biomarkers of ADHD have been uncovered to date ${ }^{7,8,70,71}$. However, studies showed results consistent with our findings: (i) impaired responses to oxidative stress have been observed in blood samples of ADHD patients, along with a relationship between low levels of pyridoxine and ADHD; ${ }^{47,58,71,72}$ (ii) despite inconsistent results regarding tryptophan pathway changes in the blood of ADHD children ${ }^{61,62}$, lower serum levels of tryptophan and related metabolites have been reported in adult patients ${ }^{73}$, in line with the association between altered tryptophan and the ADHD-like behaviors exhibited by $\operatorname{dogs}^{60}$, and as we observed in SHR/NCrl rats. Taken together, this further support the interest of exploring distinct peripheral matrices and metabolic pathways related to energy metabolism, oxidative stress, vitamins, and amino acids in the search for biomarkers of ADHD.

Questioning the relevance of potential peripheral metabolites of interest in ADHD, animal models represent an interesting opportunity to have access to both central and peripheral compartments. In addition to common differences observed at the scale of metabolic pathways between the brain and periphery of SHR/NCrl rats, we also detected metabolites that were significantly modified in the brain and periphery between SHR/NCrl rats and controls. These metabolites were related to energy metabolism and oxidative stress reinforcing the interest of investigating these metabolic pathways in peripheral compartments in the search of metabolic signature of ADHD. Significant correlations between the brain and urine variations of malonate and glucuronate were also specifically detected in SHR/NCrl rats. Such a relationship is of a great interest as these results emphasize the relevance to look at the whole individual, using cerebral but also peripheral matrices to better understand the biological modifications underlying pathological behaviors.

\section{Conclusion}

Fifteen years ago, Russell and colleagues proposed the so-called "Neuroenergetic hypothesis" of ADHD ${ }^{52}$. This hypothesis suggested alterations in neuronal transmissions due to deficits in energy supply to explain momentto-moment fluctuations in task performance, which is a common feature of $\mathrm{ADHD}^{52}$. Our results are consistent 
with this hypothesis and give new insights into the molecular mechanisms underlying such energetic dysfunction. Based on the limited access to brain metabolites in humans, the use of animal models to better understand the molecular mechanisms underlying ADHD pathogenesis remains an important avenue of research. The presence of such metabolomic modifications both in the brain and in the peripheral compartments, further support the relevance to look at these common metabolites and related metabolic pathways in the search of potential clinical ADHD biomarkers.

\section{Acknowledgements}

This study was funded by the INSERM, the Tours University and the Hyper Super TDAH France association (https://www.tdah-france.fr/). C.D. was the recipient of a PhD fellowship by the French Ministry of Higher Education, Research and Innovation. We would like to thank Florence Paillard for editing the manuscript.

\section{Conflict of interest}

The authors declare no competing interests.

\section{Publisher's note}

Springer Nature remains neutral with regard to jurisdictional claims in published maps and institutional affiliations.

Supplementary information The online version contains supplementary material available at https://doi.org/10.1038/s41398-021-01344-4.

Received: 8 September 2020 Revised: 18 March 2021 Accepted: 30 March 2021

Published online: 22 April 2021

\section{References}

1. Polanczyk, G. V. et al. ADHD prevalence estimates across three decades: an updated systematic review and meta-regression analysis. Int. J. Epidemiol. 43, 434-442 (2014).

2. Simon, V. et al. Prevalence and correlates of adult attention-deficit hyperactivity disorder: meta-analysis. Br. J. Psychiatry 194, 204-211 (2009).

3. Ramos-Quiroga, J. A. et al. Addressing the lack of studies in attention-deficit/ hyperactivity disorder in adults. Expert Rev. Neurother. 14, 553-567 (2014).

4. Faraone, S. V. Attention deficit hyperactivity disorder and premature death. Lancet 385, 2132-2133 (2015).

5. Jensen, P. S. et al. ADHD comorbidity findings from the MTA study: comparing comorbid subgroups. J. Am. Acad. Child Adolesc. Psychiatry 40, 147-158 (2001).

6. Humer, E., Probst, T. \& Pieh, C. Metabolomics in psychiatric disorders: what we learn from animal models. Metabolites 10, 106-125 (2020).

7. Faraone, S. V., Bonvicini, C. \& Scassellati, C. Biomarkers in the diagnosis of ADHD-promising directions. Curr. Psychiatry Rep. 16, 497 (2014).

8. Wang, L. J. et al. Novel plasma metabolite markers of attention-deficit/ hyperactivity disorder identified using high-performance chemical isotope labelling-based liquid chromatography-mass spectrometry. World J. Biol. Psychiatry 21, 1-10 (2020).

9. Kristal, B. S. et al. Metabolomics in the study of aging and caloric restriction. Methods Mol. Biol. 371, 393-409 (2007).

10. Bizot, J. C. et al. Methylphenidate reduces impulsive behaviour in juvenile Wistar rats, but not in adult Wistar, SHR and WKY rats. Psychopharmacology 193, 215-223 (2007).

11. Sagvolden, T. et al. The spontaneously hypertensive rat model of ADHD-the importance of selecting the appropriate reference strain. Neuropharmacology 57, 619-626 (2009).

12. Meneses, A. et al. Spontaneously hypertensive rat (SHR) as an animal model for ADHD: a short overview. Rev. Neurosci. 22, 365-371 (2011).
13. Russell, V. A. Overview of animal models of attention deficit hyperactivity disorder (ADHD). Curr. Protoc. Neurosci. 54, 9.35.1-9.35.25 (2011).

14. Sagvolden, T. \& Johansen, E. B. Rat models of ADHD. Curr. Top. Behav. Neurosci. 9, 301-315 (2012).

15. Bayless, D. W., Perez, M. C. \& Daniel, J. M. Comparison of the validity of the use of the spontaneously hypertensive rat as a model of attention deficit hyperactivity disorder in males and females. Behav. Brain Res. 286, 85-92 (2015).

16. Dieme, B. et al. Workflow methodology for rat brain metabolome exploration using NMR, LC-MS and GC-MS analytical platforms. J. Pharm. Biomed. Anal. 142, 270-278 (2017).

17. Bitar, T. et al. Identification of metabolic pathway disturbances using multimodal metabolomics in autistic disorders in a Middle Eastern population. $J$. Pharm. Biomed. Anal. 152, 57-65 (2018).

18. Chong, J., Wishart, D. S. \& Xia, J. Using MetaboAnalyst 4.0 for comprehensive and integrative metabolomics data analysis. Curr. Protoc. Bioinformatics 68, e86 (2019).

19. van den Bergh, F. S. et al. Spontaneously hypertensive rats do not predict symptoms of attention-deficit hyperactivity disorder. Pharmacol. Biochem. Behav. 83, 380-390 (2006).

20. Peres, F. F. et al. A schizophrenia-like behavioral trait in the SHR model: applying confirmatory factor analysis as a new statistical tool. Prog. Neuropsychopharmacol. Biol. Psychiatry 85, 16-22 (2018).

21. Aparicio, C. F. et al. Spontaneously hypertensive (SHR) rats choose more impulsively than Wistar-Kyoto (WKY) rats on a delay discounting task. Behav. Brain Res. 364, 480-493 (2019).

22. Leffa, D. T. et al. Systematic review and meta-analysis of the behavioral effects of methylphenidate in the spontaneously hypertensive rat model of attentiondeficit/hyperactivity disorder. Neurosci. Biobehav. Rev. 100, 166-179 (2019).

23. Niigaki, S. T. et al. Young spontaneously hypertensive rats (SHRs) display prodromal schizophrenia-like behavioral abnormalities. Prog. Neuropsychopharmacol. Biol. Psychiatry 90, 169-176 (2019).

24. Calderon-Zamora, L. et al. Changes in expression of orphan receptors GPR99 and GPR107 during the development and establishment of hypertension in spontaneously hypertensive rats. J. Recept. Signal. Transduct. Res. 29, 1-8 (2020).

25. Weise, G. et al. Transplantation of cryopreserved human umbilical cord blood mononuclear cells does not induce sustained recovery after experimental stroke in spontaneously hypertensive rats. J. Cereb. Blood Flow. Metab. 34, e1-9 (2014).

26. Onuh, J. O. \& Aliani, M. Metabolomics profiling in hypertension and blood pressure regulation: a review. Clin. Hypertens. 26, 23 (2020).

27. Coleman, M. et al. A preliminary study of the effect of pyridoxine administration in a subgroup of hyperkinetic children: a double-blind crossover comparison with methylphenidate. Biol. Psychiatry 14, 741-751 (1979).

28. Brenner, A. The effects of megadoses of selected B complex vitamins on children with hyperkinesis: controlled studies with long-term follow-up. J. Learn Disabil. 15, 258-264 (1982).

29. Dhir, S. et al. Neurological, psychiatric, and biochemical aspects of thiamine deficiency in children and adults. Front. Psychiatry 10, 207 (2019).

30. Sun, D. et al. Quantitative proteome of medulla oblongata in spontaneously hypertensive rats. J. Proteome Res. 12, 390-395 (2013).

31. Dimatelis, J. J. et al. Impaired energy metabolism and disturbed dopamine and glutamate signalling in the striatum and prefrontal cortex of the spontaneously hypertensive rat model of attention-deficit hyperactivity disorder. $J$. Mol. Neurosci. 56, 696-707 (2015).

32. Mirandola, S. R. et al. Methylmalonate inhibits succinate-supported oxygen consumption by interfering with mitochondrial succinate uptake. J. Inherit. Metab. Dis. 31, 44-54 (2008).

33. Amaral, A. U. et al. Ethylmalonic acid impairs brain mitochondrial succinate and malate transport. Mol. Genet. Metab. 105, 84-90 (2012).

34. Leffa, D. T. et al. Increased oxidative parameters and decreased cytokine levels in an animal model of attention-deficit/hyperactivity disorder. Neurochem. Res. 42, 3084-3092 (2017).

35. Reckelhoff, J. F., Romero, D. G. \& Yanes, L. L. Cardozo, sex, oxidative stress, and hypertension: insights from animal models. Physiology (Bethesda) 34, 178-188 (2019).

36. Bulut, M. et al. Malondialdehyde levels in adult attention-deficit hyperactivity disorder. J. Psychiatry Neurosci. 32, 435-438 (2007).

37. Guney, E. et al. Attention deficit hyperactivity disorder and oxidative stress: a short term follow up study. Psychiatry Res. 229, 310-317 (2015). 
38. Zhu, B., Gusdon, A. M. \& Qu, S. Weight or metabolism: which deserve more attention in obesity?. Ann. Transl. Med. 6, S127 (2018).

39. Corona, J. C. Role of oxidative stress and neuroinflammation in attentiondeficit/hyperactivity disorder. Antioxidants (Basel) 9, 1039-1056 (2020).

40. Schuck, P. F. et al. Brain and muscle redox imbalance elicited by acute ethylmalonic acid administration. PLoS ONE 10, e0126606 (2015).

41. Bordone, M. P. et al. The energetic brain - a review from students to students. J. Neurochem. 151, 139-165 (2019).

42. Fagundes, A. O. et al. Chronic administration of methylphenidate activates mitochondrial respiratory chain in brain of young rats. Int. J. Dev. Neurosci. 25 47-51 (2007).

43. Traina, G. The neurobiology of acetyl-L-carnitine. Front. Biosci. (Landmark Ed.) 21, 1314-1329 (2016)

44. Nehlig, A. Brain uptake and metabolism of ketone bodies in animal models. Prostaglandins Leukot. Essent. Fatty Acids 70, 265-275 (2004).

45. Pierre, K. \& Pellerin, L. Monocarboxylate transporters in the central nervous system: distribution, regulation and function. J. Neurochem. 94, 1-14 (2005).

46. Hertz, L. \& Kala, G. Energy metabolism in brain cells: effects of elevated ammonia concentrations. Metab. Brain Dis. 22, 199-218 (2007).

47. Joseph, N. et al. Oxidative stress and ADHD: a meta-analysis. J. Atten. Disord. 19, 915-924 (2015).

48. Guzman, M. \& Blazquez, C. Ketone body synthesis in the brain: possible neuroprotective effects. Prostaglandins Leukot. Essent. Fatty Acids 70, 287-292 (2004).

49. Harris, C. \& Hansen, J. M. Oxidative stress, thiols, and redox profiles. Methods Mol. Biol. 889, 325-346 (2012).

50. Popa-Wagner, A. et al. ROS and brain diseases: the good, the bad, and the ugly. Oxid. Med. Cell Longev. 2013, 963520 (2013).

51. Moy, L. Y., Wang, S. P. \& Sonsalla, P. K. Mitochondrial stress-induced dopamine efflux and neuronal damage by malonate involves the dopamine transporter. J. Pharmacol. Exp. Ther. 320, 747-756 (2007).

52. Russell, V. A. et al. Response variability in Attention-Deficit/Hyperactivity Disorder: a neuronal and glial energetics hypothesis. Behav. Brain Funct. 2, 30 (2006).

53. Killeen, P. R., Russell, V. A. \& Sergeant, J. A. A behavioral neuroenergetics theory of ADHD. Neurosci. Biobehav. Rev. 37, 625-657 (2013).

54. Killeen, P. R. Models of attention-deficit hyperactivity disorder. Behav. Process. 162, 205-214 (2019).

55. Purper-Ouakil, D. et al. Neurobiology of attention deficit/hyperactivity disorder. Pediatr. Res 69, 69R-76R (2011).

56. Cortese, S. The neurobiology and genetics of Attention-Deficit/Hyperactivity Disorder (ADHD): what every clinician should know. Eur. J. Paediatr. Neurol. 16 422-433 (2012).
57. Faraone, S. V. et al. Attention-deficit/hyperactivity disorder. Nat. Rev. Dis. Prim. 1, 15020 (2015).

58. Dolina, S. et al. Attention-deficit hyperactivity disorder (ADHD) as a pyridoxinedependent condition: urinary diagnostic biomarkers. Med. Hypotheses $\mathbf{8 2}$, 111-116 (2014).

59. Landaas, E. T. et al. Vitamin levels in adults with ADHD. BJPsych Open 2 377-384 (2016)

60. Altun, $\mathrm{H}$. et al. Homocysteine, pyridoxine, folate and vitamin B12 levels in children with attention deficit hyperactivity disorder. Psychiatr. Danub $\mathbf{3 0}$ 310-316 (2018).

61. Kennedy, D. O. B vitamins and the brain: mechanisms, dose and efficacy-a review. Nutrients $\mathbf{8}, 68$ (2016).

62. Grobecker, $\mathrm{H}$. et al. Peripheral and central catecholaminergic neurons in genetic and experimental hypertension in rats. Clin. Sci. Mol. Med. Suppl. 3, 377s-380s (1976)

63. Wocial, B. et al. Tissue catecholamine concentrations in spontaneously hypertensive rats. Acta Physiol. Pol. 28, 303-312 (1977).

64. Saavedra, J. M. Adrenaline levels in brain stem nuclei and effects of a PNMT inhibitor on spontaneously hypertensive rats. Brain Res. 166, 283-292 (1979).

65. Chalmers, J. P. et al. Brainstem PNMT neurons and experimental hypertension in the rat. Clin. Exp. Hypertens. A 6, 243-258 (1984).

66. Louis, W. J. et al. Neurotransmitters and neuropeptides in blood pressure regulation in the spontaneously hypertensive rat. Can. J. Physiol. Pharmacol. 65, 1633-1637 (1987).

67. Grandbois, J. et al. Phenylethanolamine N-methyltransferase gene expression in adrenergic neurons of spontaneously hypertensive rats. Neurosci. Lett. $\mathbf{6 3 5}$, 103-110 (2016).

68. Sevigny C. P. et al. in Stress: Neuroendocrinology and Neurobiology, (ed. G. Fink), Academic Press, Chapter 3, 29-37 (2017).

69. An, M. \& Gao, Y. Urinary biomarkers of brain diseases. Genomics Proteomics Bioinformatics 13, 345-354 (2015).

70. Scassellati, C. et al. Biomarkers and attention-deficit/hyperactivity disorder: a systematic review and meta-analyses. J. Am. Acad. Child Adolesc. Psychiatry 51, 1003-1019.e20 (2012)

71. Bonvicini, C., Faraone, S. V. \& Scassellati, C. Common and specific genes and peripheral biomarkers in children and adults with attention-deficit/hyperactivity disorder. World J. Biol. Psychiatry 19, 80-100 (2018).

72. Ozcan, O. et al. Plasma leptin, adiponectin, neuropeptide $Y$ levels in drug naive children with ADHD. J. Atten. Disord. 22, 896-900 (2018).

73. Aarsland, T. I. et al. Serum concentrations of kynurenines in adult patients with attention-deficit hyperactivity disorder (ADHD): a case-control study. Behav. Brain Funct. 11, 36 (2015). 